\title{
Phase-resolved PIV measurements of the flow between a pair of corotating disks in a cylindrical enclosure
}

\author{
Y.S. Tsai ${ }^{\mathrm{a}}$, Y.M. Chang ${ }^{\mathrm{a}}$, Y.J. Chang ${ }^{\mathrm{b}}$, Y.M. Chen ${ }^{\mathrm{a}, *}$ \\ ${ }^{a}$ Department of Mechanical Engineering, National Taiwan University, Taipei, Taiwan 106, ROC \\ ${ }^{\mathrm{b}}$ Department of Management of Information System/Institute of Biomedical Engineering and Material Science, Chungtai Institute of \\ Health Science and Technology, Taichung, Taiwan 406, ROC
}

Received 13 July 2005; accepted 4 August 2006

Available online 9 October 2006

\begin{abstract}
This paper presents the measurements of the flow in the space between an enclosed corotating disk pair using particle image velocimetry (PIV) and laser doppler velocimetry (LDV). LDV gives the time history of velocity for time-domain analysis, while PIV provides the spatial distribution of the instantaneous velocity. A flow visualization technique displaying the concentration distribution of seeding particles was also employed to visualize the flow patterns. Experiments were conducted on the interdisk midplane with a Reynolds number of $5.25 \times 10^{5}$. Based on the LDV measured rotating frequency of the vortices around the hub, the phase-resolved PIV measurements were achieved, and a rotating reference coordinate system was employed to represent the flow patterns. The phase-resolved measurements reveal that the circumferential flow velocity oscillates periodically in both the inner and outer regions but in opposite trends. Based on the phase averaged data, the contributions of the periodic and random motions to the Reynolds stresses were evaluated, and the spatial distributions of the periodic Reynolds stresses were displayed. It is found that, the local rotation of the fluid induced by the deformation of the inner region contribute to a significant portion of the momentum transport.
\end{abstract}

(C) 2006 Elsevier Ltd. All rights reserved.

Keywords: PIV; LDV; Corotating disks; Phase-resolved measurements

\section{Introduction}

The unobstructed flow between a pair of disks corotating in a cylindrical enclosure (Fig. 1) is one of the classical problems in fluid mechanics and involves complex physics. The flow phenomena are also of practical importance in the design of turbines, compressors, disk storage devices and other rotating machinery because of the commonality in the geometrical configuration.

The first analysis motivated by this flow configuration might be attributed to Stewartson (1957), who obtained the cross-stream (side-view, as the $r-z$ plane illustrated in Fig. 1) flow characteristics consisting of two counter-rotating toroidal vortices. The cross-stream flow patterns were characterized much later in the calculations of Chang et al. (1989), as well as in the analysis of Schuler et al. (1990).

\footnotetext{
*Corresponding author. Tel.: + 886233662730 ; fax: + 886223634701 .

E-mail address: ymchen@ntu.edu.tw (Y.M. Chen).
} 


\begin{tabular}{|c|c|c|c|}
\hline \multicolumn{2}{|c|}{ Nomenclature } & $\overline{\mathbf{u}}, \tilde{\mathbf{u}}, \mathbf{u}^{\prime}$ & global mean, periodic and random compo- \\
\hline$a$ & clearance between disk and shroud & $\tilde{\mathbf{u}} \tilde{\mathbf{u}}$ & periodic component of Reynolds stress \\
\hline$D$ & height of spacer ring & $\mathbf{u}^{\prime} \mathbf{u}^{\prime}$ & random component of Reynolds stress \\
\hline$f$ & primary flow frequency & $\mathbf{u}_{r}$ & radial flow velocity \\
\hline$M$ & number of phase samples & $\mathbf{u}_{\theta}$ & circumferential flow velocity \\
\hline$N$ & $\begin{array}{l}\text { number of samples acquired at a constant } \\
\text { phase }\end{array}$ & $u_{\theta}^{*}$ & $\begin{array}{l}\text { normalized circumferential velocity defined } \\
\text { as } \mathbf{u}_{\theta} /(\boldsymbol{\Omega} \times \mathbf{r})\end{array}$ \\
\hline$R_{1}$ & radius of hub & $\bar{u}_{\theta}^{*}$ & normalized mean circumferential velocity \\
\hline$R_{2}$ & radius of disk & & defined as $\overline{\mathbf{u}}_{\theta} /(\boldsymbol{\Omega} \times \mathbf{r})$ \\
\hline $\mathrm{Re}$ & Reynolds number defined as $\Omega R_{2}^{2} / v$ & $z$ & axial coordinate \\
\hline$r$ & radial coordinate & $z^{*}$ & normalized axial distance defined as $z / D$ \\
\hline$r^{*}$ & $\begin{array}{l}\text { normalized radial distance defined as }(r- \\
\left.R_{1}\right) /\left(R_{2}-R_{1}\right)\end{array}$ & $\stackrel{\zeta}{\zeta}, \tilde{\zeta}$ & $\begin{array}{l}\text { vorticity } \\
\text { global mean and periodic component of } \zeta\end{array}$ \\
\hline $\mathbf{r}$ & radial distance from the hub center & $\theta$ & azimuthal coordinate \\
\hline$S$ & $\begin{array}{l}\text { normalized axial distance between disks } \\
\text { defined as } D / R_{2}\end{array}$ & & $\begin{array}{l}\text { kinematic viscosity } \\
\text { phase delay }\end{array}$ \\
\hline$t$ & time coordinate & $\Omega, \boldsymbol{\Omega}$ & disk rotating speed and velocity \\
\hline $\mathbf{u}$ & flow velocity variable & & \\
\hline
\end{tabular}

The early visualization experiments of Lennemann (1974) and Kaneko et al. (1977) have provided a qualitative insight concerning the flow on the midplane between corotating disks (plane-view, as the $r-\theta$ plane illustrated in Fig. 1). They observed a polygon region of laminar flow near the hub, rotating at roughly $80 \%$ of the disk rotating speed. The laminar core was found to be surrounded by more turbulent flow structures.

Abrahamson et al. $(1989,1991)$ also studied the flow on the interdisk midplane $(r-\theta)$ and observed three distinct regions of flow that they referred to as an "inner region" in solid body rotation surrounding the hub, an "outer region" containing axially aligned vortical structures, and a "shroud boundary layer region" between the outer region and the enclosure wall. The authors postulated that the outer region vortices give shape to the polygonal inner region. In their work, a hot film probe was also used to measure the circumferential velocity and the power spectra. By dividing the actual frequencies of spectra by $75 \%$ of the disks rotating speed, the results were found showing peaks in the energy at integral normalized frequencies.

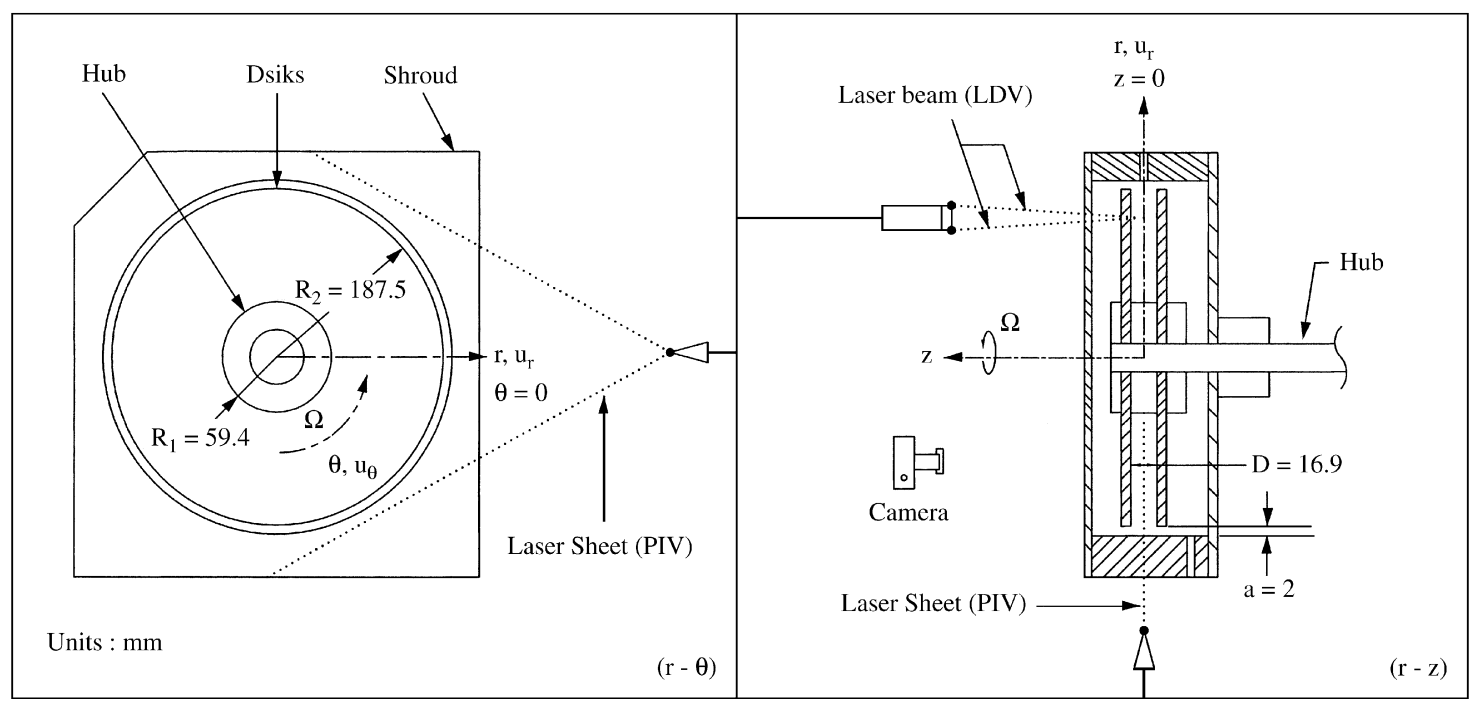

Fig. 1. Test model, coordinate system and the optical arrangement. 
The flow velocity measurements were also made using Laser Doppler Velocimetry (LDV) (Schuler et al., 1990), and both the mean and r.m.s. circumferential velocity profiles were revealed. The maxima in the radial profiles of the r.m.s. circumferential velocity were attributed to the oscillations produced by the passage of the axially aligned vortices. Schuler et al. also presented a comprehensive analysis based on scaling considerations. The results (Fig. 2) show that the cross-stream flow field $(r-z)$ is composed of five distinct regions: (i) a region of strongly sheared fluid near the enclosure wall; (ii) a core region of potential flow, characterized by moderate velocity gradients in the radial direction and negligible gradients in the axial direction; (iii) an axially aligned detached shear layer that allows the transition between the motion in the potential core and in the solid body region; (iv) a region essentially in solid body rotation near the hub; and (v) a pair of Ekman layers developing on the facing surfaces of the two disks. The appearance of the flow structures on the interdisk midplane $(r-\theta)$ was deduced to be related to the oscillations of the cross-stream flow $(r-z)$.

Humphrey et al. (1991) reviewed most of the relevant prior work and concluded that the polygon shape of the inner region is associated with the axially aligned vorticity, responsible for circumferentially periodic radially directed inflows and outflows. Subsequently, Humphrey and Gor (1993) visualized the cross-stream flow $(r-z)$ in an experimental apparatus similar to those used by Schuler et al. (1990), and observed a pair of counter-rotating toroidal vortices at the location corresponding to that of the region ii noted by Schuler et al. (1990). The authors postulated a link between the axially aligned vortices and the instability of the cross-stream vortex pair. Quantitative evidence was then provided by the numerical calculations of Humphrey et al. (1995), and the onset of instability was found to be associated with a 2-D to 3-D transition in flow patterns.

More recently, Iglesias and Humphrey (1998) investigated the effects of Reynolds number (Re) and disk spacing $(S)$ on the flow patterns numerically. The simulation results showed that, as the Reynolds number varies, variations might occur in the qualitative structure of the solutions for certain values of the parameters. Herrero et al. (1999a, b, 2002) also investigated numerically the effects of geometry and buoyancy on the flow structure. A qualitative bifurcation diagram was established to elaborate the transition of flow modes, and three different families of cross-stream flow $(r-z)$ were defined. At a low value of the $(\operatorname{Re}, S)$ pair, the counter-rotating vortices show symmetry with respect to the interdisk midplane, with patterns similar to those illustrated in Fig. 2. At a higher Re or a larger $S$, the 2-D steady flow (denoted as regime I) may experience a pitchfork or a Hopf bifurcation to 3-D flow types. The pitchfork bifurcation may result in a flow that is no longer symmetric with respect to the interdisk midplane (regime III), while the Hopf bifurcation may lead to a flow that displays shift and reflect symmetry (regime II). Besides, the radially directed outflows and inflows affecting the structure and dynamics of the solid body region were also mentioned. The bifurcation phenomena were further investigated by Randriamampianina et al. (2001).

Summarizing the prior studies, at a sufficiently high rotating speed, the symmetric characteristic of the cross-stream flow $(r-z)$ motion is lost and the interface demarcating the solid body region (iv) acquires a polygon shape. On the other hand, the interdisk midplane flow $(r-\theta)$ reveals special patterns, characterized by circumferentially periodic, axially aligned vortices, whose formation is associated with the instability of the cross-stream flow $(r-z)$. However, the previous understanding is to a greater extent based on point-wise measurements. The analysis based on the measured data may only apply in a time-averaged sense. The velocity or vorticity fields were determined in the above-mentioned numerical work; however, a real-time whole-field measurement still seems lacking. Besides, the axially aligned vortical

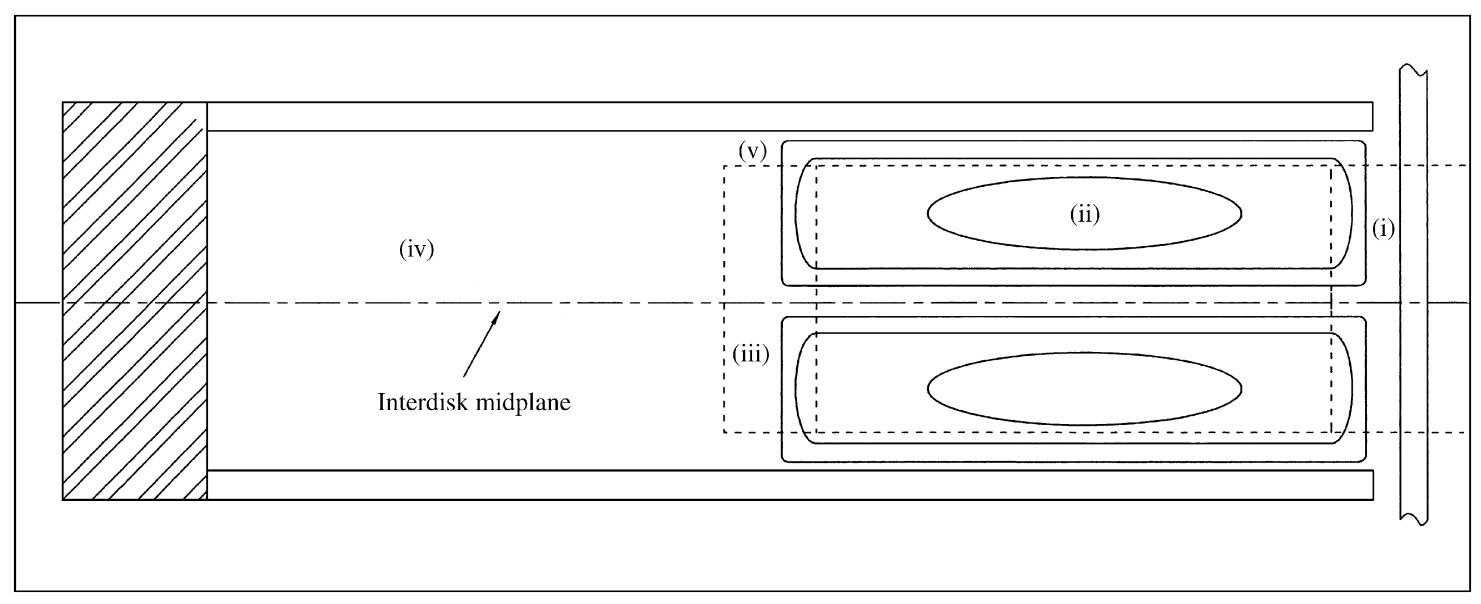

Fig. 2. Flow structure ( $r-z$ plane) proposed by Schuler et al. (1990). 
structures were still hard to recognize in flow visualizations. Consequently, there is no complete experimental data to structurally and quantitatively determine the flow patterns.

In order to explore this flow configuration in more detail, experimental work was carried out using both particle image velocimetry (PIV) and LDV. With the use of PIV, the $r-\theta$ midplane flow structures were quantified with a digital camera. LDV could record the time history of the velocity for time-domain analysis. Furthermore, based on the time period information detected by LDV, the phase-resolved PIV measurements without an extra phase identifier were conducted. The measurement results could give the time evolution of the spatial distribution of velocity. Besides, a flow visualization technique displaying the distribution of seeding particles was also employed to verify the measured flow information. The present effort seeks to reconstruct the flow field and to analyze the motion of the circumferentially periodic flow structures.

\section{Experimental apparatus}

\subsection{Test model and experimental condition}

The flow configuration investigated here consists of a fixed cylindrical enclosure containing two coaxial disks attached to and corotating with a hub, as shown in Fig. 1. Two rigid disks are concentrically clamped on a hub. A spacer ring of height $D=16.9 \mathrm{~mm}$ is mounted concentrically with the disks to separate them in the spindle-axis direction (z-direction). The ratio of the axial separation to disk radius is $0.09\left(S=0.09=D / R_{2}\right)$ since it has been widely studied in the prior literature (Herrero et al., 1999b). In order to facilitate the measurements, water was used as the working fluid, so that a high Reynolds number could be achieved at a relatively low speed of rotation. The measurements were conducted on the midplane between the two disks $\left(z^{*}=0=z / D\right)$.

Experiments were conducted at a Reynolds number of $5.25 \times 10^{5}$, where $\operatorname{Re}=\Omega R_{2}^{2} / v$, based on the disks' rotating speed $\Omega$, radius $R_{2}$, and the water kinematic viscosity $v$. At this Re with $S=0.09$, the flow condition corresponds to the 3-D symmetry-breaking flow type [regime III in the notation of Herrero et al. (1999a)] and may display most of the characteristics reported previously. Abrahamson et al. (1991) also mentioned that, with Re greater than $2.5 \times 10^{5}$, the axially aligned vortical structures are easy to visualize and may maintain their identities for many revolutions of the disks. Hence, the present experimental condition would be helpful for investigating the typical corotating disk flow.

\subsection{PIV system}

PIV is a nonintrusive technique for simultaneously measuring the whole flow field. The fluid was seeded with tracer particles and the $r-\theta$ plane under investigation was illuminated. A camera recorded the images of particles illuminated by the pulsed light sheets. During the camera shutter time, the measured plane was illuminated twice by two separate lasers, and the positions of particles for two laser pulses were taken on the same frame. Velocity information was obtained from the double-exposed PIV image by quantifying the average spatial shift of the particles during the illumination time interval by an autocorrelation algorithm. The detailed theory of PIV was introduced by Adrian (1991).

In the present study, double-pulsed Nd:Yag lasers (TSI Model Y150, with a maximum energy output of $150 \mathrm{~mJ} /$ pulse at $532 \mathrm{~nm}$, and a repetition rate of $10 \mathrm{~Hz}$ for each) were used to supply pulsed laser sheets for illuminating the measured plane. The polycrystalline particles with approximate diameter of $30 \mu \mathrm{m}$ and density of $0.98 \mathrm{~g} / \mathrm{mL}$ were used as the PIV tracers. The particles were injected by an injector inserted through the enclosure, and their mechanical properties were checked using the equation given by Raffel et al. (1998). A Canon EOS D30 digital camera whose shutter is capable of being triggered by an electronic signal was used to capture the PIV images. The twin Nd:Yag lasers and the camera were controlled by a synchronizer. The image resolution of camera was set at $2160 \times 1440$, making the camera resolution limiting.

The PIV image was transferred to a computer for gray scaling. The processed image with 256 gray levels was then divided into several small interrogation areas for quantifying the average particle displacement within each of them. The images were divided into $64 \times 64$ pixel interrogation areas, and 50\% overlap grids were employed for the PIV image processing. Commercial software (TSI Insight) was used to process the PIV image and to compute the velocity vectors through the autocorrelation algorithm. Since the vortex structures or velocity reversals would not be presented in the laboratory frame, the velocity field could thus be calculated without directional ambiguity from a single double-exposed PIV image. 
The accuracy of velocity measurements was estimated, depending on the ability to determine the displacement of a particle (Adrian, 1991). Here, a parabolic interpolation method was used to search for the peak of the autocorrelation function with sub-pixel accuracy, and hence the uncertainty of the vector-processing algorithm was reduced to 0.03 pixels. The time interval between two pulsed lasers was chosen to give the particle displacement of not less than 6 pixels, to keep a relative error below $0.5 \%$. For a typical flow velocity of $1.5 \mathrm{~m} / \mathrm{s}$, this error corresponds to a velocity error of $\pm 0.75 \mathrm{~cm} / \mathrm{s}$. However, there are a number of other aspects in PIV measurements that can affect the accuracy of measurements due to imperfections in the apparatus, the particular PIV parameters used, and the nature of the flow being measured. In order to ensure the reliability of PIV measurements, a calibration based on the comparison of the measurement results of LDV and PIV was applied. Here, the time average of the instantaneous velocity measured by PIV was compared with the mean velocity measured by LDV. The deviation between two velocity data was found to be within $3 \%$.

\subsection{LDV system}

LDV measures the flow velocity by the Doppler shift in the light scattered from the seeding particles. A twocomponent fiber-optic LDV system worked in a forward scattering mode during the measurements. The optical module consists of a TSI Model 9201 Cold-burst Multicolor Beam Separator, four Model 9271 couplers, an Argon-ion laser (Coherent Innova 90, $2 \mathrm{~W}$ ), a probe with front collimating lens of $350 \mathrm{~mm}$ focal length, a single-mode polarizationpreserving fiber, a Bragg cell, two pinhole filters and two photomultiplier optics sections. The associated electronics include two photomultipliers, two electronic frequency shifters, three TSI 1990C counter processors, a TSI-MI990 buffer interface, and a Yokogawa DL-1200A oscilloscope.

Here, the LDV system was operated with a data rate maintained at $2000 \mathrm{~Hz}$ to ensure the quality of the measurements. For the LDV measurements, the particle diameter must be smaller than the fringe spacing produced by the optical system. Hence, the magnesium oxide particles with approximate diameter of $1 \mu \mathrm{m}$ and density of $3.56 \mathrm{~g} / \mathrm{mL}$ were used. The diameter and density of particles result in a Stokes number of $10^{-6}$ in the water simulator. More than 30,000 samples at each point obtained by LDV at the data rate of $2000 \mathrm{~Hz}$ were used to calculate the flow velocity. The bias for calculating the global mean velocity was corrected by the method provided by McLaughlin and Tiederman (1973). The maximum and typical uncertainties of mean velocity were estimated to be $5.7 \%$ and $3.1 \%$, respectively.

\section{Experimental methodology}

\subsection{Phase-resolved PIV measurements}

Following the work of Reynolds and Hussain (1972), the time-varying velocity variable $\mathbf{u}(x, t)$ can be viewed formally as a combination of a global mean component $\overline{\mathbf{u}}(x)$, a periodic mean component $\tilde{\mathbf{u}}(x, t)$, and a residual random component $\mathbf{u}^{\prime}(x, t)$. By definition, the total velocity variable is the sum

$$
\mathbf{u}(x, t)=\overline{\mathbf{u}}(x)+\tilde{\mathbf{u}}(x, t)+\mathbf{u}^{\prime}(x, t) .
$$

In order to obtain the respective components, the data need to be phase-averaged. The periodic mean and random components may then be defined as

$$
\begin{aligned}
\tilde{\mathbf{u}}(x, t) & =\langle\mathbf{u}(x, t)\rangle-\overline{\mathbf{u}}(x), \\
\mathbf{u}^{\prime}(x, t) & =\mathbf{u}(x, t)-\langle\mathbf{u}(x, t)\rangle,
\end{aligned}
$$

where \langle\rangle is the average at a constant phase. Here, phase-averaging was realized by taking an average over the conditional samples at a given phase

$$
\langle\mathbf{u}(x, \tau)\rangle_{m}=\frac{1}{N_{m}} \sum_{i=1}^{N_{m}} \mathbf{u}\left(x, t_{i}+\tau_{m}\right),
$$

where $N_{m}$ is the number of samples acquired at phase $\tau_{m}$ from the reference time $t_{i}$. The global mean values were obtained from the mean of the phase-averaged quantities, using the following expression:

$$
\overline{\mathbf{u}}(x)=\frac{1}{M} \sum_{m=1}^{M}\langle\mathbf{u}(x, \tau)\rangle_{m},
$$

where $M$ is the number of phases. 
In the present study, the frequency determined from the LDV measured data suggests that there are three circumferentially periodic vortical structures rotating at about $75 \%$ of the rotating speed of the disks, and the rotating period is found to be $0.6 \mathrm{~s}(1 / 0.75 \Omega)$. Once the sampling interval of the camera was adjusted to be equal to the rotating period, the flow motion would be phase-locked. Here, the synchronizer generated the trigger train for triggering both the lasers and the camera. The phase jitter in $\tau_{m}$ was estimated from the difference between each cycle and the average over a predetermined number $(N=15)$ of cycles. Due to the synchronization underlying the phase maintenance, the effect of phase jitter which is the time integral of frequency jitter is neglected.

Since the vortical structures are distributed evenly in the $r-\theta$ midplane and are circumferentially periodic, by controlling the frame rate of the camera the phase-resolved PIV measurements without an extra phase identifier can be achieved. Here, the velocity fields at six consecutive phases during $1 / 3$ of a period are presented in order to investigate the time evolution of the flow. In other words, $M=18$ was used, and therefore the cycle was sampled at $20^{\circ}$ intervals. However, the camera is not capable of taking the subsequent images with such a short interval. To achieve this sampling interval, several runs were made, and the trigger time was shifted $0.6 \mathrm{~s}$ for each run. Hence, the phase delay was set at $380^{\circ}$ but not $20^{\circ}$.

The phase-averaged vector fields can be produced by conditionally sampling data on the basis of the precise phase. The convergence of the velocity was examined as a function of the number of the velocity fields in an ensemble. For the flow structures which are strongly periodic in time, only 10 samples at each point and phase may be needed for convergence of the phase-averaged data (Perry et al., 1980). Here, preliminary PIV investigations suggested that $N=15$ samples are sufficient for convergence of the phase-averaged velocity fields without phase jitter superimposed on them. A maximum mean value error of $5 \%$ of the maximum velocity with $90 \%$ confidence level was considered as a converged velocity field at a constant phase. In the present experiments, the phase-averaged velocity field was obtained using 15 samples, while the global mean velocity field was obtained using 270 samples. The resulting statistical uncertainties are typically $\pm 1.1 \%$ for the global mean velocity and within $\pm 7.3 \%$ for the three components of periodic Reynolds stresses.

Besides, the time interval in phase-resolved PIV measurements was also calibrated. In order to estimate the trigger delay between sending the electronic trigger signal and the opening of the camera shutter, the calibration work was carried out by imaging a marked point consecutively, with the frame rate the same as the rotating rate of the point. By examining the point positions in sequential pictures, the uncertainty was estimated to be within $1 \%$.

\subsection{Flow visualization method}

In order to visualize the flow structure on the $r-\theta$ plane, a flow visualization technique displaying the particle distribution was used. The PIV tracer particles mentioned above were seeded into the flow and illuminated by a light sheet generated by the laser of the PIV system. The particles were injected adjacent to the hub by an injector inserted through the enclosure. Near the hub where the fluid was in solid body rotation, the particles accumulated and reflected the light sheet, causing a bright region. Away from the hub, where the flow mixing rates were high, the few particles penetrating from the bright region were diluted rapidly. A useful consequence of this method is that the different particle concentrations help to distinguish between regions of different flow types.

\section{Results and discussion}

\subsection{Mean velocity and power spectra}

Fig. 3 shows the radial profile of normalized mean circumferential velocity component measured by LDV. The circumferential velocity on the midplane is normalized by rotating velocity $(\boldsymbol{\Omega} \times r)$ of the disks, which is represented by the horizontal dashed line. From the hub edge to the enclosure wall, the profile can be roughly divided into two parts. First, from $r^{*}=0$ to 0.40 , the normalized velocity remains 1 , coinciding with the rotating disks. The previous measurements conducted at different Re (Schuler et al., 1990) or $S$ (Abrahamson et al., 1989) might also reveal similar velocity profiles with horizontal sections, when their results were plotted in dimensionless coordinates (Fig. 7(b)). The region in which the mean circumferential velocity coincides with that of the disks was hence called the inner region or solid-body-rotation region. Second, from $r^{*}=0.40$ to about 0.98 , the normalized velocity simply declines from 1 to about 0.54 . This region was considered to be dominated by large vortical structures with significant vorticity aligned in the axial direction, which were almost evenly distributed around a circle approximately $2 / 3$ of the distance between the hub and the enclosure wall. Although many experimental works have been conducted to reconstruct the $r-\theta$ midplane 


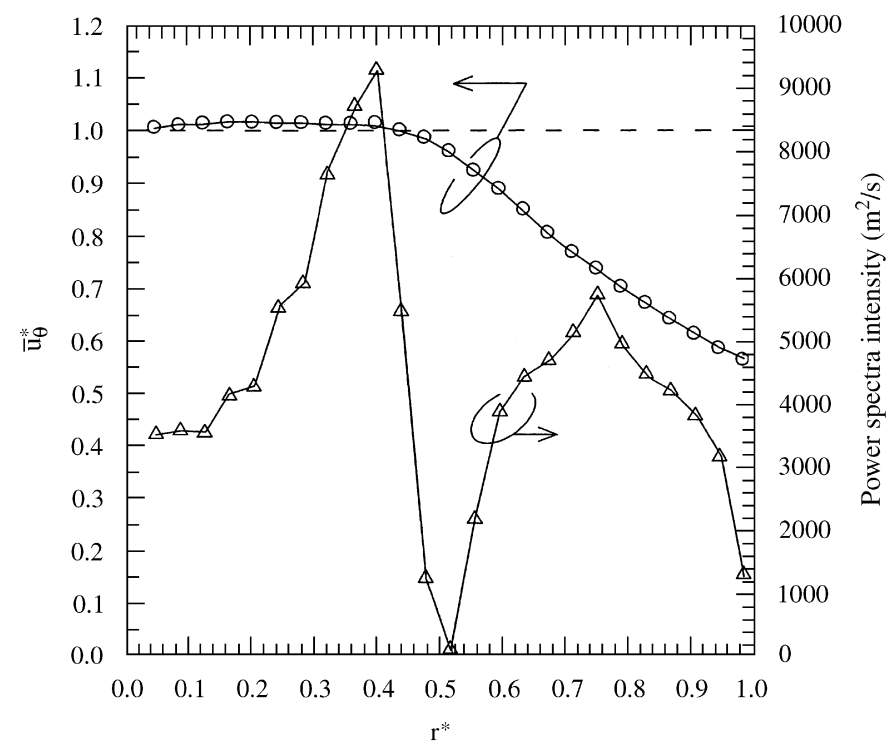

Fig. 3. Radial distribution of normalized mean circumferential velocity and the spectral intensity of circumferential velocity at $f=5.01 \mathrm{~Hz}\left(S=0.09, \mathrm{Re}=5.25 \times 10^{5}\right)$.
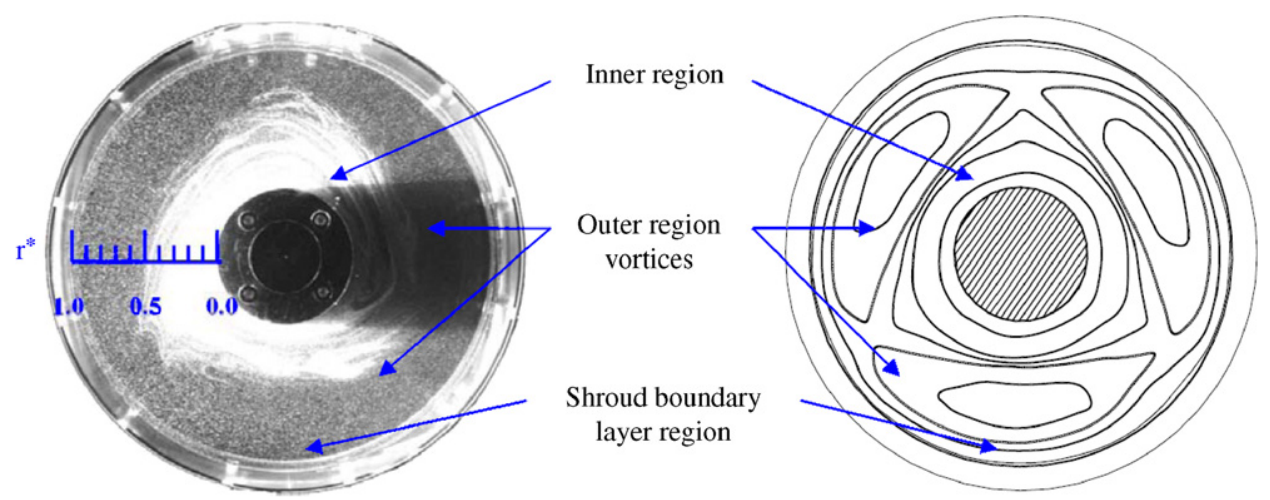

Fig. 4. Visualization image and schematic drawing of the $r-\theta$ plane flow field $\left(S=0.09, \operatorname{Re}=5.25 \times 10^{5}\right)$.

flow, the vortical structures were hard to recognize in flow visualizations and only the qualitative sketches were reported previously.

To investigate the flow structures and serve as the verification of the present measurements, flow visualization work was also conducted. Fig. 4 was taken at an instant before the particles injected near the hub dispersed vigorously. The flow visualization on the $r-\theta$ midplane revealed clearly two parts, which roughly corresponded to the division mentioned above. The inner region near the hub looked bright, since the particles retained there reflected the illuminating light strongly. The bright inner region rotated around the hub and was shaped as a triangle. Since the number of vortical structures would correspond to the number of polygonal sides of the inner region (Abrahamson et al., 1989), the regular triangle inner region reflects that three large-scale vortical structures are distributed evenly in the outer region.

The partition between the inner and outer regions was evidenced by the fact that the particles accumulated most in the inner region for several minutes. The complex fluid motion in the outer region may cause the disordered distribution of particles. As a result, a small number of particles penetrating from the inner region showed a diffuse, grainy distribution, resulting in a spotted outer region. Although the large vortical structures are still hardly identifiable, the contrast between the bright and spotted regions helps to clarify the shape and extent of the inner region. It is found that the radius of the inscribed circle of the triangle intersects the radial coordinate axis at about $r^{*}=0.40$, while the radius of circumscribed circle intersects the axis at about $r^{*}=0.60$. Although not shown here, the $r-\theta$ plane flow fields at 
different axial positions $\left(z^{*}=0.3-0.7\right)$ showed similar flow patterns. This suggests that the measurement conducted on the interdisk midplane is representative of the $r-\theta$ plane flow.

The time history of velocity recorded by LDV was also used to conduct a power spectral analysis, which was considered to be helpful for understanding the periodicity of the flow. The results show that the power spectra at different measurement positions along the radial direction all converge at the same primary frequency of $5.01 \mathrm{~Hz}$. Abrahamson et al. (1989) mentioned that, if the primary flow frequency $(f)$ is normalized by $75 \%$ of the rotating speed of the disks $(\Omega)$, the value obtained would correspond to the number of vortical structures $(N=f /(0.75 \Omega))$. Substituting the present values $(f=5.01 \mathrm{~Hz}, \Omega=133$ r.p.m. $\approx 2.22 \mathrm{~Hz})$ into the above yields that the number of vortical structures is three, which corresponds to the flow visualization results. According to the above-mentioned algorithm, the primary flow frequency would be the frequency at which all the three vortical structures pass by the LDV probe successively. When the primary frequency is divided by three, the value obtained $(1.67 \mathrm{~Hz})$ denotes the frequency at which each single vortical structure passes by the probe. In other words, the vortical structures rotate at about $75 \%(1.67 / 2.22)$ of the disk speed of rotation.

Fig. 3 also shows a possibility of finding two local maxima of the spectral intensity. The first maximum is located at $r^{*}=0.40$, while the second is located at $r^{*}=0.75$, reflecting that the circumferential velocity is more energetic at these two positions. It deserves to be noted that the spectral intensity is almost zero at $r^{*}=0.52$, indicating that the circumferential velocity does not oscillate at this position. Schuler et al. (1990) measured the radial profile of r.m.s. circumferential velocity and also obtained a similar feature that they referred to as the max-min-max pattern. The authors suggested that the velocity oscillations most probably result from the periodic passage of axially aligned vortical structures. The extremely low r.m.s. velocity, for which the reason was not known previously, may be attributed to a special flow phenomenon, as discussed in Section 4.4.

Summarizing the above, the flow structures were found rotating periodically at a specific frequency, and the positions at which the most energetic flow motion occurs were also discovered. However, the vortical structures have not yet been identified in the flow visualization results. Besides, the mean velocity profile can represent neither the velocity fluctuation revealed by the spectral intensity nor the variations in the radial extent of the inner region revealed by flow visualization. In order to solve these problems, phase-resolved PIV measurements were employed to give an impression of the flow characteristics over an entire region of the flow field.

\subsection{Streamline patterns}

Fig. 5 shows the PIV measured velocity and streamline fields. Fig. 5(a) was generated with a stationary reference frame with respect to the laboratory, while Figs. 5(b) and (c) were generated with the reference frame moving at $0.75 \boldsymbol{\Omega} \times \mathbf{r}$ and coinciding with the rotation of flow structures. The frame rate of the camera was used to check the rotating reference velocity. Given the frame rate of $75 \%$ of the disk rotating speed and the reference velocity of $0.75 \Omega \times \mathbf{r}$, the vortical structures in sequential pictures appear almost at the same radial and circumferential location, i.e., the rotation of vortical structures is essentially frozen. Different reference velocity values and frame rates were also tested. Higher or lower reference velocities would make the center of the vortical structure deviate from the radial position mentioned above. On the other hand, the sequence of pictures at a higher frame rate showed a vortical structure moving backward in the circumferential direction, while those at a lower frame rate showed the structure moving forward. The reference velocity $(0.75 \boldsymbol{\Omega} \times \mathbf{r})$ resulting from the present analysis corresponds to the value reported by Abrahamson et al. (1989).

Since the frequency at which each vortical structure rotates is known, the phase-resolved PIV measurement is possible. Based on the discussions in the preceding paragraph, the rotating period of each single vortical structure was confirmed to be $0.6 \mathrm{~s}(1 / 0.75 \Omega)$. This period was then divided into equal parts to serve as the sampling interval for the camera. As a result, the passage of a vortical structure lasting for $1 / 3$ of its rotating period was revealed in Fig. 6(1)-(6). Due to the periodicity of the structure, Fig. 6(7), which is not shown, would show flow patterns similar to those in Fig. 6(1), although the vortical structure within it is a subsequent one.

As shown in Fig. 6, the inner flow region surrounding the hub acquires the shape of a polygon. The area that surrounds the inner region is the outer region containing circumferentially periodic vortical structures. In order to clarify the flow structures, the critical points of flow patterns that include the center point $(\mathbf{\Lambda})$ of the vortical structure and the saddle point $(x)$ are also marked in Fig. 6 . It is found that, the center point coincides with the middle of the side of the polygon, while the saddle point coincides with the vertex of inner region. In addition, between the outer region vortical structures and the enclosure wall, a thin flow region is also found. It corresponds to the shroud boundary layer region mentioned by Abrahamson et al. (1991), but has not been presented in prior flow visualizations. 


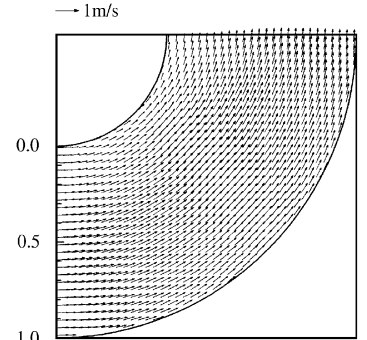

(a)

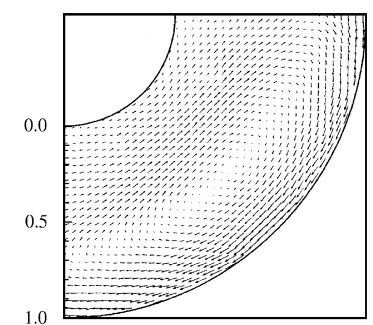

(b)

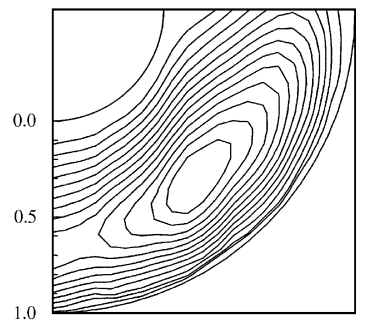

(c)

Fig. 5. (a) Vector field in the laboratory frame, (b) vector field, and (c) streamline patterns in the rotating reference frame $(S=0.09$, $\left.\operatorname{Re}=5.25 \times 10^{5}\right)$
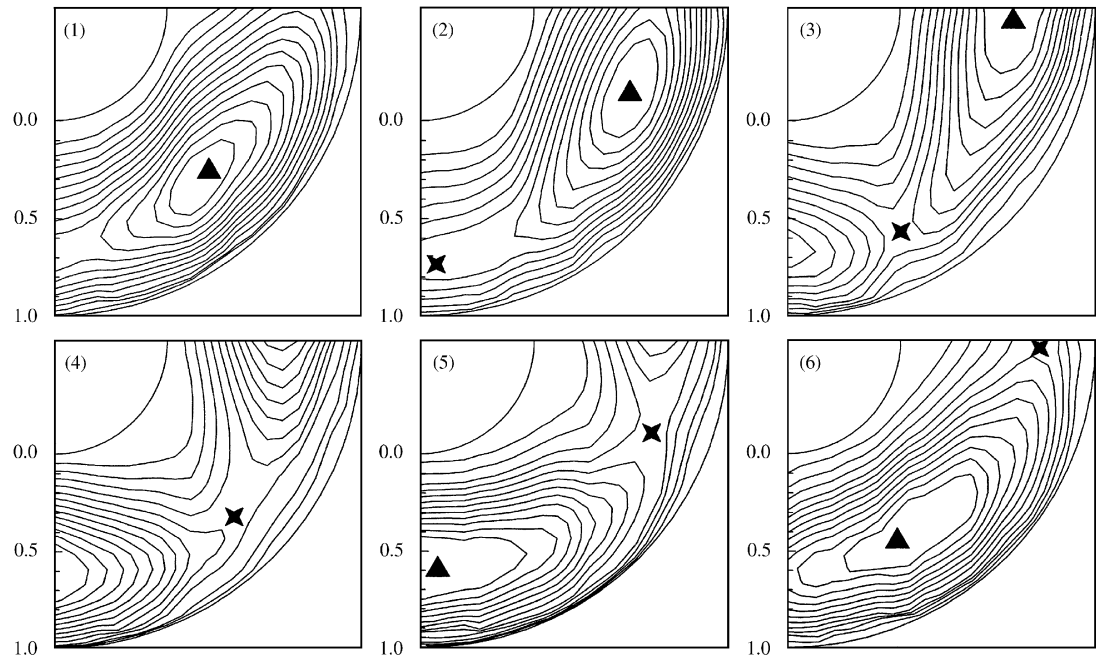

Fig. 6. Streamline patterns in the rotating reference frame at consecutive phases (rotating reference frame) for $S=0.09$ and $\operatorname{Re}=5.25 \times 10^{5}$.

The present PIV streamline patterns further show that the radial extent of this region also varies with time and circumferential position.

\subsection{Phase-resolved velocity}

To describe the flow motion, the instantaneous velocity profiles at the consecutive phases corresponding to Fig. 6 were also explored. The instantaneous circumferential velocity profiles along the horizontal axis $\left(\theta=0^{\circ}\right)$ are shown in Fig. 7(a). Here, the circumferential velocity in lab reference frame is also normalized by $\boldsymbol{\Omega} \times \mathbf{r}$. The normalized instantaneous circumferential velocity at the consecutive phases exhibits different radial profiles corresponding to the passage of the vortical structure. The contrast between Figs. 6 and 7(a) reveals that when the vortical structure approaches the sampling axis $\left(\theta=0^{\circ}\right)$, the radial extent of inner region shrinks gradually, and the normalized circumferential velocity near the hub also becomes increasingly larger. Until the center point arrives, the normalized circumferential velocity achieves a peak value larger than the disk speed of rotation. Conversely, when a vortical structure leaves, the velocity near the hub becomes lower and lower and the radial extent of the inner region expands gradually. In prior studies, the inner region was qualitatively found moving in solid body rotation, but with small amplitude cyclic motion, in response to the passing train of the vortical structures (Abrahamson et al., 1989). Here, the instantaneous circumferential velocity profiles at the consecutive phases quantitatively reveal this slight flow motion. 


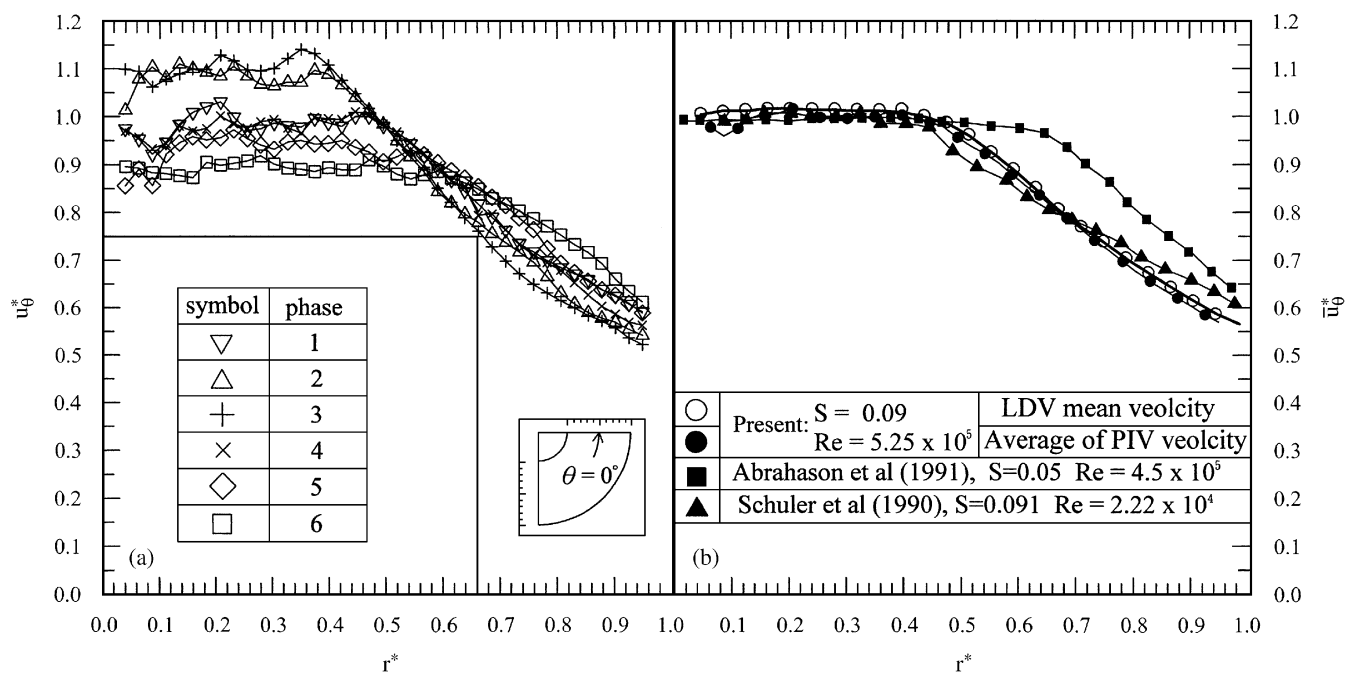

Fig. 7. (a) Radial profiles of normalized circumferential velocity at the consecutive phases corresponding to Fig. 6; (b) the global mean normalized circumferential velocity $\left(S=0.09, \mathrm{Re}=5.25 \times 10^{5}\right)$.

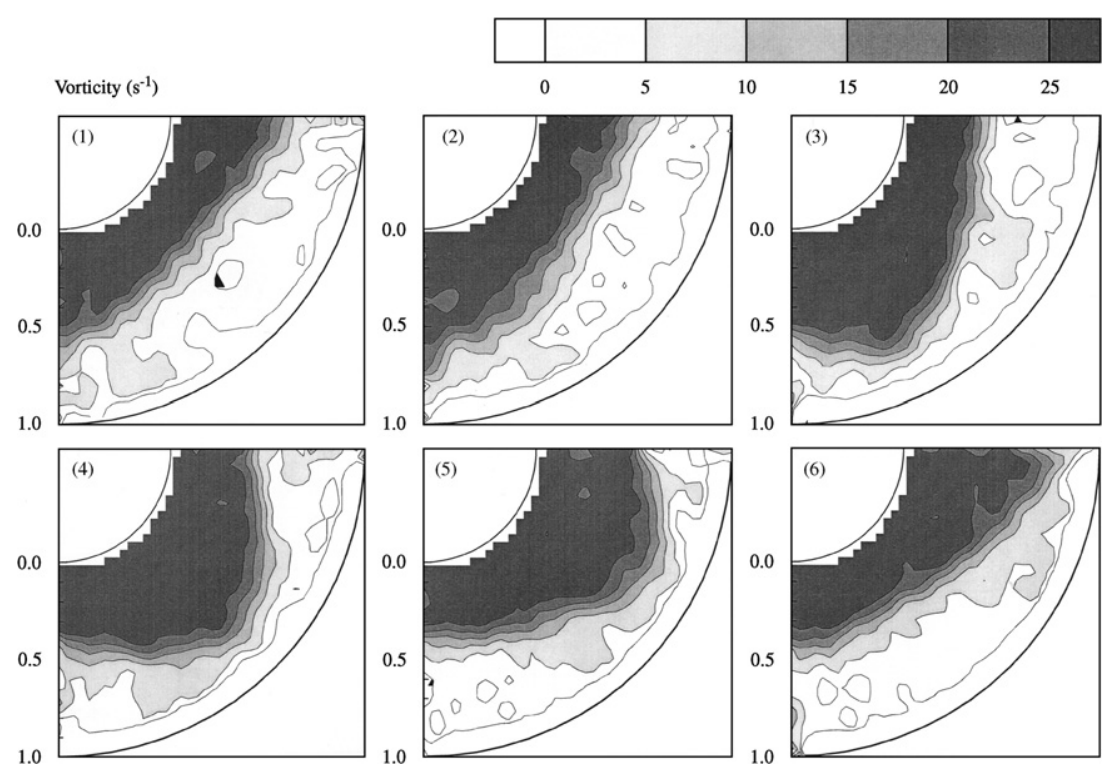

Fig. 8. Vorticity field at consecutive phases corresponding to Fig. $6\left(S=0.09, \operatorname{Re}=5.25 \times 10^{5}\right)$.

As shown in Fig. 7(a), the circumferential velocity in the inner region rises and falls with time. A similar oscillation phenomenon is also found in the outer region, but the trend is opposite to that of the inner region. The profiles at different phases intersect at about $r^{*}=0.52$, indicating that the circumferential velocity does not oscillate there. This corresponds to the spectral feature mentioned above. Since the oscillation of velocity is strongly periodic, the periodic variations in velocity would be offset due to the averaging effect intrinsic to the method of point-wise measurement. Hence, the normalized mean circumferential velocity shown in Fig. 7(b) cannot explain the distribution of spectral intensity. Besides, the radial position where the normalized mean velocity starts to decline cannot accurately represent the interface between inner and outer regions. On the other hand, as shown in Fig. 7(b), the average of the PIV measured velocity profiles approximates the mean velocity profile measured by LDV. 


\subsection{Periodic fluctuating vorticity and velocity fields}

In Fig. 7(b), the global mean normalized circumferential velocity, which is assumed to be independent of time, remains equal to 1 from the hub edge to $r^{*}=0.4$ and then simply declines thereafter. Corresponding to the velocity profile, the global mean vorticity $(\bar{\zeta})$ would be a function of radial distance only. The inner region could be viewed as a circular region of nearly constant and high vorticity with an obvious vorticity gradient at its edge.

Traditionally, the large-scale flow structures can be deduced by examining the vorticity field at a constant phase,

$$
\langle\zeta\rangle=\frac{1}{r}\left[\frac{\partial\left(r\left\langle u_{\theta}\right\rangle\right)}{\partial r}-\frac{\partial\left\langle u_{r}\right\rangle}{\partial \theta}\right]
$$

In order to investigate the large-scale flow motion, the PIV measured data in the lab reference frame were phase averaged according to Eq. (4). As shown in Fig. 8, $\langle\zeta\rangle$ retains large positive values up to a polygonal curve corresponding to the interface between the inner and outer regions shown in Fig. 6 . This reflects that the fluid in the inner region is subject to a sinusoidal radial displacement. In prior studies, the axially aligned vortical structures were regarded as the foci of intensified axial component vorticity. Here, no negative vorticity focus corresponding to the clockwise vortices is observed, while only the positive vorticity corresponding to the counterclockwise rotation around the hub is found. It may be due to the global mean circumferential flow strongly dominating the flow field. Hence, to indicate the periodic fluctuating flow motions, the phase averaged vorticity $\langle\zeta\rangle$ was split into two components and the periodic fluctuating component $(\tilde{\zeta})$ with respect to the global mean was displayed. In Fig. 9 , the periodic fluctuating vorticity $(\tilde{\zeta})$ fields are shown in contour plots with the magnitude represented by the gray scale, and the solid and dashed lines denote the positive and negative values, respectively. As shown, the positive periodic fluctuating vorticity foci coincide with the saddle points shown in Fig. 6, while the negative ones coincide with the center points.

Previously, the formation of the circumferentially periodic, axially aligned vortices was considered to be associated with the instability of the cross-stream $(r-z)$ flow (Humphrey and Gor, 1993; Humphrey et al., 1995). Herrero et al. (1999a) further provided a qualitative bifurcation diagram and found that, at sufficiently large values of $\operatorname{Re}$ or $S$, the 2D axisymmetric cross-stream flow (regime I) may experience a pitchfork bifurcation toward another 2-D flow that is no longer symmetric with respect to the interdisk midplane. The symmetry-breaking 2-D flow further evolves toward a wavy symmetry-breaking 3-D flow (regime III). This 2-D to 3-D evolution coincides with the appearance of radial inflows and outflows giving the polygonal shape to the inner region. The outflow jets induce the vertex of the polygon by pulling the inner region away from the hub, while the inflow jets may flatten the side of the inner region by forcing it inward. It seems reasonable to suggest that this circumferential chain of alternating inflows and outflows may induce the sinusoidal deformation of the inner region and hence the periodic fluctuating vorticity as shown in Fig. 9.

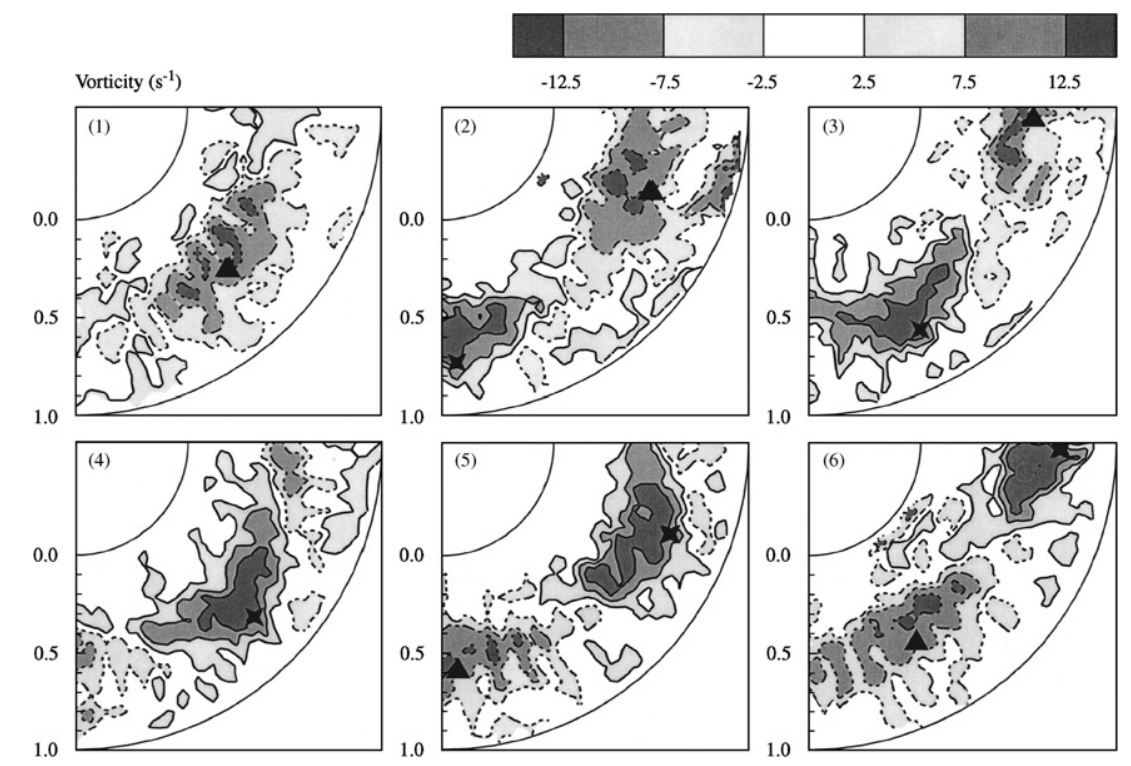

Fig. 9. Periodic component of the vorticity at the consecutive phases corresponding to Fig. $6\left(S=0.09, \operatorname{Re}=5.25 \times 10^{5}\right)$. 

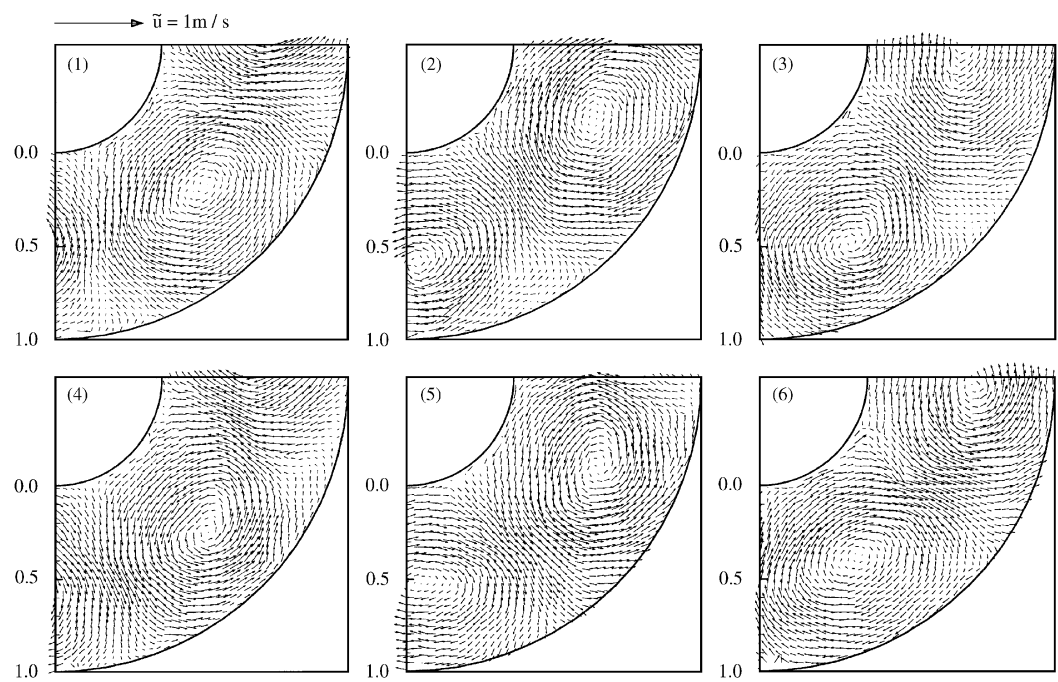

Fig. 10. Periodic component of the velocity at consecutive phases corresponding to Fig. $6\left(S=0.09, \operatorname{Re}=5.25 \times 10^{5}\right)$.

According to the invertibility principle (Hoskins et al., 1985), the fluctuating vorticity may induce the fluctuating velocity field. The periodic fluctuating velocity ( $\tilde{\mathbf{u}})$ fields with respect to the global mean are shown in Fig. 10. There could be seen a chain of alternating clockwise and anticlockwise fluctuating vortices, the centers of which are evenly distributed around a circle approximately $1 / 2$ of the distance between the hub and the shroud. The center of anticlockwise vortex coincides with the vertex of the polygon, while that of clockwise vortex coincides with the middle of the side of the polygon. Since there is no velocity fluctuation at the centers of the fluctuating vortices, the circumferential velocity does not vary at $r^{*}=0.52$, resulting in the extremely low spectral intensity there. The radial position of the fluctuating vortex center is where the un-normalized mean circumferential velocity achieves its maximum value. Schuler et al. (1990) also mentioned that the minimum of the r.m.s. velocity occurs at the location where the mean circumferential velocity attain its maximum value.

This chain of periodically fluctuating vortices may affect the flow dynamics. The radial component of the periodic fluctuating velocity may advect the fluid parcel outward left of the positive fluctuating vorticity, and inward left of the negative fluctuating vorticity (Holton, 1997). As a result, the fluctuating vorticity patterns would propagate circumferentially backward relative to the global mean flow. However, due to the dominance of the strong circumferential flow, the vorticity patterns would still propagate circumferentially forward, but with a propagation velocity slower than the mean circumferential velocity. This may explain why the propagation of the polygonal interface between the inner and outer regions is slower than the velocity of flow in the inner region.

\subsection{Momentum transport}

In this section, the velocity in the lab reference frame was triply decomposed, and investigation was made of the distributions and values of the Reynolds stresses. When an ensemble of velocity data is considered at a given phase, the average of this ensemble may describe the repeatable large-scale flow motion, ũ. In addition, for turbulence modeling, the fluctuations due to the small-scale random turbulence superimposed on the large-scale flow structures, or due to the fact that the large-scale structure is not itself the same in every sample, should also be considered. The fluctuations with respect to the mean value at a fixed phase would be considered as the random component, $\mathbf{u}^{\prime}$.

Before quantifying the amounts of the two components of the total Reynolds stresses, a preliminary analysis on the percentages for the ratios of random to total stresses was conducted. According to Cantwell and Coles (1983), the total Reynolds stresses were obtained by adding the global averaged periodic and random stresses:

$$
\overline{\tilde{\mathbf{u}} \tilde{\mathbf{u}}}+\overline{\left\langle\mathbf{u}^{\prime} \mathbf{u}^{\prime}\right\rangle}=\frac{1}{M} \sum_{m=1}^{M}\left(\tilde{\mathbf{u}} \tilde{\mathbf{u}}+\left\langle\mathbf{u}^{\prime} \mathbf{u}^{\prime}\right\rangle\right) .
$$

Here, these two components are themselves an average over one rotating cycle of measurements. Due to the time averaging, the global mean fields would be regarded as function of radial distance only, and were hence presented as 
radial profiles. As shown in Fig. 11, the radial profile of $\overline{\tilde{\mathbf{u}}_{\theta} \tilde{\mathbf{u}}_{\theta}}+\overline{\left\langle\mathbf{u}_{\theta}^{\prime} \mathbf{u}_{\theta}^{\prime}\right\rangle}$ shows the max-min-max pattern similar to that of spectral intensity, while the maximum of $\overline{\tilde{\mathbf{u}}_{r} \tilde{\mathbf{u}}_{r}}+\overline{\left\langle\mathbf{u}_{r}^{\prime} \mathbf{u}_{r}^{\prime}\right\rangle}$ is located at about $r^{*}=0.52$. The values of random circumferential and radial stresses are both much lower than those of the total stresses. Exceptions occur only at $r^{*}=0.52$ for $\overline{\mathbf{u}_{\theta} \tilde{\mathbf{u}}_{\theta}}+\overline{\left\langle\mathbf{u}_{\theta}^{\prime} \mathbf{u}_{\theta}^{\prime}\right\rangle}$, and at the locations adjacent to hub and enclosure for $\overline{\tilde{\mathbf{u}}_{r} \tilde{\mathbf{u}}_{r}}+\overline{\left\langle\mathbf{u}_{r}^{\prime} \mathbf{u}_{r}^{\prime}\right\rangle}$, since the values of periodic stresses are extremely low there. Since the values of random stresses along the radial profiles are extremely low, the radial profiles of periodic stresses approximate those of total stresses very well, and hence are not displayed. The low percentages of random stresses suggest that the variations in shape and position of the flow structures at each phase are considerably low, indicating that the flow is not highly turbulent. Since the large-scale structure contributes largely to the total stresses and for the sake of brevity, only the periodic component of Reynolds stresses is emphasized here.

In Figs. 12-14, the periodic Reynolds stresses were resolved as functions of position and phase. The values of three different periodic Reynolds stresses which include circumferential $\left(\tilde{\mathbf{u}}_{\theta} \tilde{\mathbf{u}}_{\theta}\right)$, radial $\left(\tilde{\mathbf{u}}_{r}, \tilde{\mathbf{u}}_{r}\right)$, and shear $\left(\tilde{\mathbf{u}}_{r}, \tilde{\mathbf{u}}_{\theta}\right)$ stresses are normalized by $R_{2}^{2} \Omega^{2}$. The peaks in $\tilde{\mathbf{u}}_{\theta} \tilde{\mathbf{u}}_{\theta}$ on either radial side of $r^{*}=0.52$, with $\tilde{\mathbf{u}}_{\theta}$ positive for one peak and negative for the other, generated by the periodic motion with respect to the global mean are shown in Fig. 12. The maximum normalized value of $\tilde{\mathbf{u}}_{\theta} \tilde{\mathbf{u}}_{\theta}$ is found to be 0.012 at about $r^{*}=0.40$ in the radial sections across the centers of fluctuating vortices. The corresponding r.m.s. velocity is about $10 \%$ of the global mean circumferential velocity. In Fig. 13, the peaks in $\tilde{\mathbf{u}}_{r} \tilde{\mathbf{u}}_{r}$ are evenly distributed around a circle of $r^{*}=0.52$ with magnitude comparable to that of $\tilde{\mathbf{u}}_{\theta} \tilde{\mathbf{u}}_{\theta}$, and correspond to positive and negative peaks in $\tilde{\mathbf{u}}_{r}$ alternately. The peaks in $\tilde{\mathbf{u}}_{r} \tilde{\mathbf{u}}_{\theta}$ shown in Fig. 14 exhibit a four-lobed clover-leaf-type distribution, which includes two positive (solid line) and two negative (dashed line) peak zones surrounding the fluctuating vortex center. The sign of $\tilde{\mathbf{u}}_{r} \tilde{\mathbf{u}}_{\theta}$ reflects the direction of momentum flux. Taking the peaks
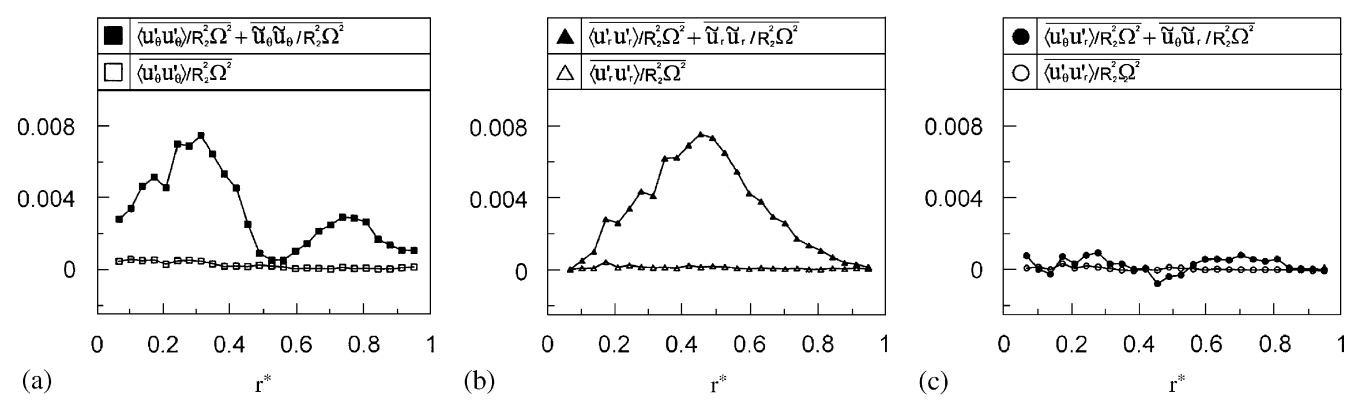

Fig. 11. Radial profiles of the global mean random and total Reynolds stresses: (a) circumferential normal, (b) radial normal, and (c) shear stresses.
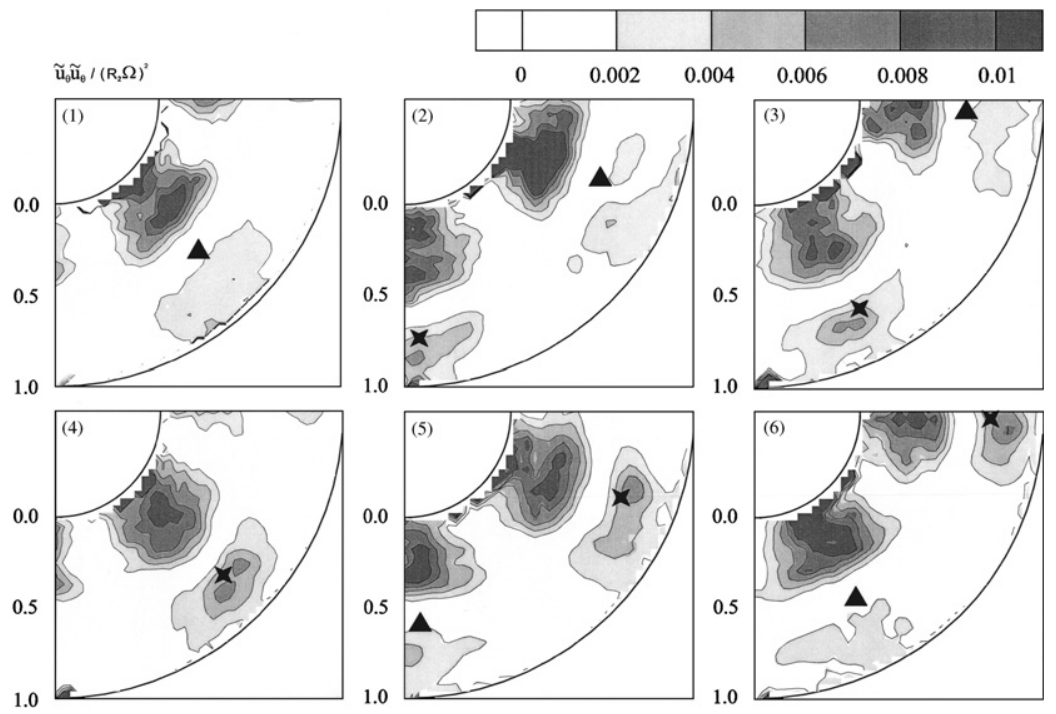

Fig. 12. Periodic component of circumferential normal Reynolds stress at consecutive phases corresponding to Fig. $6(S=0.09$, $\left.\operatorname{Re}=5.25 \times 10^{5}\right)$ 


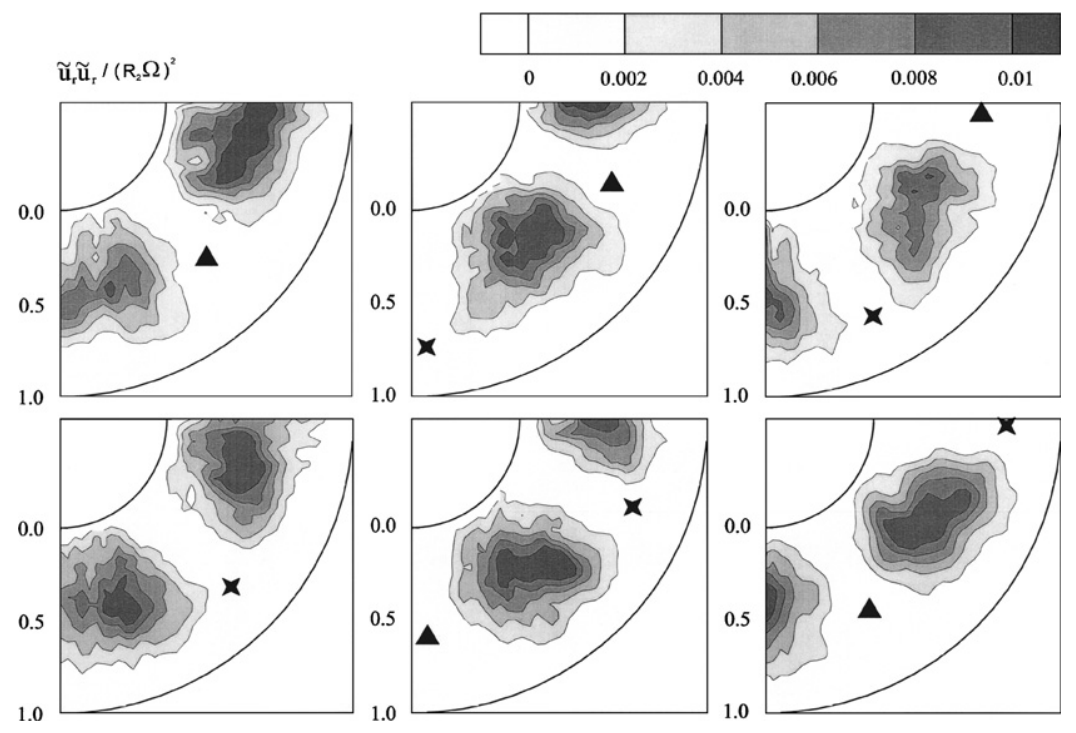

Fig. 13. Periodic component of radial normal Reynolds stress at consecutive phases corresponding to Fig. 6 ( $S=0.09$, $\left.\operatorname{Re}=5.25 \times 10^{5}\right)$
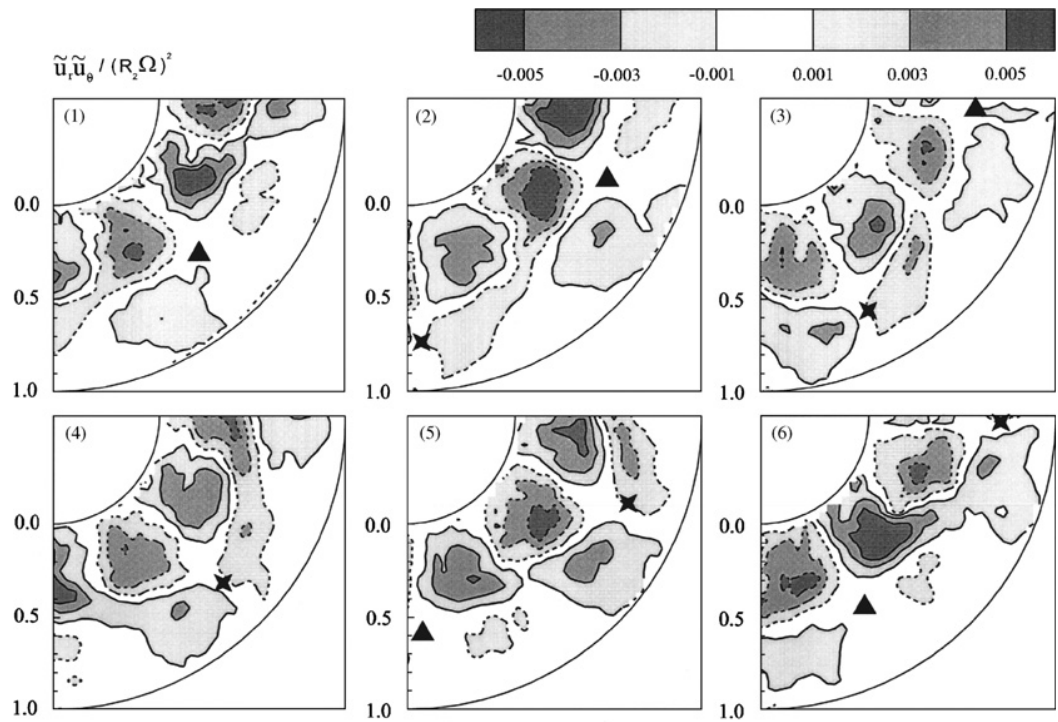

Fig. 14. Periodic component of Reynolds shear stress at consecutive phases corresponding to Fig. $6\left(S=0.09, \operatorname{Re}=5.25 \times 10^{5}\right)$.

near the hub, for example, a positive $\tilde{\mathbf{u}}_{r} \tilde{\mathbf{u}}_{\theta}$ would cause the circumferential momentum flux to deviate from the positive circumferential direction and cause it to be pushed toward the positive radial direction (outward). Conversely, a negative $\tilde{\mathbf{u}}_{r} \tilde{\mathbf{u}}_{\theta}$ denotes that a flux in the negative radial direction (inward) transfers to the positive circumferential direction. Along a radial section, the peak values of $\tilde{\mathbf{u}}_{r} \tilde{\mathbf{u}}_{\theta}$ would exhibit opposite signs with respect to $r^{*}=0.52$. However, the signs of $\tilde{\mathbf{u}}_{r} \tilde{\mathbf{u}}_{\theta}$ may alternate with the circumferential positions of the sampling section. In Fig. 11, due to the time averaging performed, the circumferentially periodic variations in $\tilde{\mathbf{u}}_{r} \tilde{\mathbf{u}}_{\theta}$ would be offset. As a result, both the values of $\overline{\left\langle\mathbf{u}_{r}^{\prime} \mathbf{u}_{\theta}^{\prime}\right\rangle}$ and $\overline{\tilde{\mathbf{u}}_{r} \tilde{\mathbf{u}}_{\theta}}+\overline{\left\langle\mathbf{u}_{r}^{\prime} \mathbf{u}_{\theta}^{\prime}\right\rangle}$ along the radial profile are extremely low.

The periodic motion is primarily a consequence of the local self-rotation induced by the above-mentioned periodic fluctuating vorticity. As a result, the stress contours are confined mostly around $r^{*}=0.52$. The arrangement of the stress contours also appears to circumferentially coincide with the vortical structures as viewed from a reference frame. 


\section{Conclusions}

PIV and LDV were applied to investigate the flow arising in the space between a pair of disks corotating in a cylindrical enclosure. Using LDV, the frequency of velocity oscillations resulting from the passage of circumferentially periodic structures was detected. Based on the frequency, phase-resolved PIV measurements were conducted and the $r-\theta$ plane flow patterns were reconstructed. Through the flow evolution at consecutive phases, the periodic flow motion was investigated. In addition to power spectra, the periodic fluctuating velocity fields and the distributions of Reynolds stresses were also presented. The major conclusions are summarized below:

(i) The phase resolved PIV measurements successfully reconstructed the flow structures at specific rotating phases. The measurement results concretely confirm that the periodic passage of axially aligned vortical structures is responsible for the primary flow frequency, which is the rotating frequency of the vortical structures around the hub multiplied by the number of structures.

(ii) In the inner region, the circumferential velocity does not coincide with the rotating disks, but rises and falls periodically. The velocity also oscillates periodically in the outer region with the trend opposite to that in the inner region.

(iii) The periodic velocity component exhibits a chain of alternating clockwise and anticlockwise fluctuating vortices. The fluctuating vortices are evenly distributed around a circle of radius approximately half of the distance between the hub and the shroud, and cause the velocity oscillations around their centers.

(iv) The ratio of periodic to total stresses reflects that the repeatable large-scale variations in the velocity are primarily responsible for the total Reynolds stresses. The periodic stresses are consequences of self-rotation of the periodic fluctuating vortices with respect to the global mean.

\section{References}

Abrahamson, S.D., Eaton, J.K., Koga, D.J., 1989. The flow between shrouded corotating disks. Physics of Fluids A1, $241-251$.

Abrahamson, S.D., Eaton, J.K., Chang, C., 1991. Flow structure in head-disk assemblies and implications for design. ASME Advances in Information Storage Systems 1, 111-132.

Adrian, R.J., 1991. Particle-imaging techniques for experimental fluid mechanics. Annual Review of Fluid Mechanics 23, $261-304$.

Cantwell, B., Coles, D., 1983. An experimental study of entrainment and transport in the turbulent near wake of a circular cylinder. Journal of Fluid Mechanics 136, 321-374.

Chang, C.J., Schuler, C.A., Humphrey, J.A.C., Greif, R., 1989. Flow and heat transfer in the space between two corotating disks in an axisymmetric enclosure. Journal of Heat Transfer 111, 625-632.

Herrero, J., Giralt, F., Humphrey, J.A.C., 1999a. Influence of the geometry on the structure of the flow between a pair of corotating disks. Physics of Fluids 11, 88-96.

Herrero, J., Giralt, F., Humphrey, J.A.C., 1999b. Non-isothermal laminar flow and heat transfer between disks corotating in a fixed enclosure. International Journal of Heat and Mass Transfer 42, 3291-3306.

Herrero, J., Humphrey, J.A.C., Giralt, F., 2002. Effect of radial clearance on corotating disk flows in fixed cylindrical enclosures. ASME Journal of Fluids Engineering 124, 719-727.

Holton, J.R., 1997. An Introduction to Dynamic Meteorology, 4th ed. Academic Press, San Diego.

Hoskins, B.J., McIntyre, M.E., Robertson, A.W., 1985. On the use and significance of isentropic potential vorticity maps. Quarterly Journal of the Royal Meteorological Society 111, 877-946.

Humphrey, J.A.C., Gor, D., 1993. Experimental observation of an unsteady detached shear layer in enclosed corotating disk flow. Physics of Fluids 5, 2438-2442.

Humphrey, J.A.C., Chang, C.J., Li, H.W., Schuler, C.A., 1991. Unobstructed and obstructed rotating disk flows: a summary review relevant to information storage systems. ASME Advances in Information Storage Systems 1, 79-110.

Humphrey, J.A.C., Schuler, C.A., Webster, D.R., 1995. Unsteady laminar flow between a pair of disks corotating in a fixed cylindrical enclosure. Physics of Fluids 7, 1225-1240.

Iglesias, I., Humphrey, J.A.C., 1998. Two- and three-dimension laminar flows between disks co-rotating in a fixed cylindrical enclosure. International Journal for Numerical Methods in Fluids 26, 581-603.

Kaneko, R., Oguchi, S., Hoshiya, K., 1977. Hydrodynamic characteristics in disk packs for magnetic storage. Review of the Electrical Communication Laboratories 25, 1325-1336.

Lennemann, E., 1974. Aerodynamic aspects of disk files. IBM Journal of Research and Development 18, 480-488.

McLaughlin, D.K., Tiederman, W.G., 1973. Biasing correction for individual realization of laser anemometer measurements in turbulent flows. Physics of Fluids 16, 2082-2088.

Perry, A.E., Lim, T.T., Chong, M.S., 1980. The instantaneous velocity fields of coherent structures in coflowing jets and wakes. Journal of Fluid Mechanics 101, 243-256. 
Raffel, M., Willert, C., Kompenhans, J., 1998. Particle Image Velocimetry: A Practical Guide. Springer, Berlin.

Randriamampianina, A., Schiestel, R., Wilson, M., 2001. Spatio-temporal behavior in an enclosed corotating disk pair. Journal of Fluid Mechanics 434, 39-64.

Reynolds, W.C., Hussain, A.K.M.F., 1972. The mechanics of an organized wave in turbulent shear flow. Part 3: Theoretical models and comparison with experiments. Journal of Fluid Mechanics 54, 263-288.

Schuler, C.A., Usry, W., Weber, B., Humphrey, J.A.C., Greif, R., 1990. On the flow in the unobstructed space between shrouded corotating disks. Physics of Fluids A2, 1760-1770.

Stewartson, K., 1957. On almost rigid rotations. Journal of Fluid Mechanics 3, 17-26, 131-144. 